\title{
Polydopamine layer formation at the liquid - gas interface
}

DOI:

10.1016/j.colsurfa.2019.123637

\section{Document Version}

Accepted author manuscript

Link to publication record in Manchester Research Explorer

\section{Citation for published version (APA):}

Milyaeva, O. Y., Bykov, A. G., Campbell, R., Loglio, G., Miller, R., \& Noskov, B. A. (2019). Polydopamine layer formation at the liquid - gas interface. Colloids and Surfaces A: Physicochemical and Engineering Aspects, 579, 123637. https://doi.org/10.1016/j.colsurfa.2019.123637

\section{Published in:}

Colloids and Surfaces A: Physicochemical and Engineering Aspects

\section{Citing this paper}

Please note that where the full-text provided on Manchester Research Explorer is the Author Accepted Manuscript or Proof version this may differ from the final Published version. If citing, it is advised that you check and use the publisher's definitive version.

\section{General rights}

Copyright and moral rights for the publications made accessible in the Research Explorer are retained by the authors and/or other copyright owners and it is a condition of accessing publications that users recognise and abide by the legal requirements associated with these rights.

\section{Takedown policy}

If you believe that this document breaches copyright please refer to the University of Manchester's Takedown Procedures [http://man.ac.uk/04Y6Bo] or contact uml.scholarlycommunications@manchester.ac.uk providing relevant details, so we can investigate your claim.

\section{open 2 access}




\title{
Polydopamine layer formation at the liquid - gas interface
}

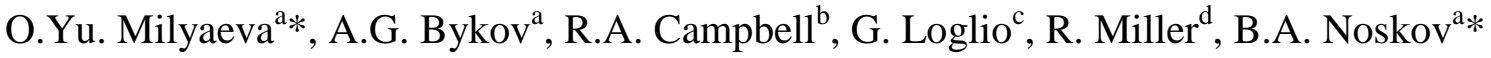 \\ ${ }^{a}$ Department of Colloid Chemistry, St. Petersburg State University, Universitetsky pr. 26, 198504 \\ St. Petersburg, Russia \\ ${ }^{b}$ Division of Pharmacy and Optometry, University of Manchester, Manchester M13 9PT, UK \\ ${ }^{c}$ Institute of Condensed Matter Chemistry and Energy Technology, CNR- ${ }^{d}$ ICMATE, Genova, Italy \\ ${ }^{d}$ Max-Planck-Institute for Colloid and Interface Science, D-14476 Golm, Germany
}

Corresponding Authors

*E-mail: borisanno@rambler.ru, o.milyaeva@spbu.ru

\begin{abstract}
The surface properties of a polydopamine layer at the air-water interface were studied by dilatational surface rheology, ellipsometry and Brewster angle microscopy (BAM). A significant increase of the dynamic surface elasticity was discovered when the concentration changed from $0.75 \mathrm{~g} / \mathrm{l}$ to $2 \mathrm{~g} / \mathrm{l}$ with the maximum value of about $60 \mathrm{mN} / \mathrm{m}$ at a concentration of $1 \mathrm{~g} / \mathrm{l}$. The obtained results indicate that the surface film consists of separate domains and the high surface elasticity is a consequence of the interactions between relatively rigid domains of the polymer film. This conclusion was confirmed by Brewster angle microscopy, which demonstrated different steps of the polydopamine film growth. Separate domains appeared at the first step while one can observe a continuous film close to equilibrium. An increase of the initial concentration led to faster polymerization and to the formation of a thicker film. The dynamic surface elasticity decreased in the concentration range from $2 \mathrm{~g} / \mathrm{l}$ to $5 \mathrm{~g} / \mathrm{l}$ when the thickness of the polymer film reached about $80 \mathrm{~nm}$. In this case the film could be destroyed in the course of deformation. The cracks in the film resulted in a decrease of the dynamic surface elasticity.
\end{abstract}

Keywords: polydopamine, polymerization, air-water interface, dilatational surface visco-elasticity, BAM, surface ellipsometry 


\section{Introduction}

Polydopamine is a prospective material for surface coating and functionalization [1-3]. Most of its applications are connected with its very high adhesive ability [4-6]. Indeed polydopamine can be fixed firmly on all types of surfaces regardless of their chemical nature [1]. Studies of the adhesive properties of polydopamine are subject to extensive works. The main applications of polydopamine as a component of composite nanomaterials in combination, for example, with graphene or metal nanoparticles, are based on this unique property [7-9]. There are also some attempts to create nanoparticles, membranes, and capsules based on this polymer [3,10]. The high biological compatibility of this compound is also of great interest because of various biomedical applications [11].

Polydopamine is usually obtained by oxidation of dopamine in its solution [10,12-14]. Dopamine is easily oxidized and undergoes spontaneous polymerization in slightly alkaline environment $[10,15,16]$. Oxygen present in air or dissolved in water acts as an oxidant. The reaction proceeds spontaneously under mild conditions when commercially available dopamine hydrochloride is dissolved in aqueous alkaline solutions[17-19]. The polymer film is formed on the liquid surface and polymer grains appear in the bulk. The thickness of the film can be controlled by changing the initial monomer concentration and time of polymerization. The thickness of the polydopamine film on the surface of the solution with a concentration of $2 \mathrm{~g} / \mathrm{l}$ can reach about $50 \mathrm{~nm}[19]$.

In spite of the relative simplicity of dopamine polymerization, the details of this process and the polymer structure are still being discussed. The earliest hypothesis suggests that polydopamine is formed in the same way as melanin in living organisms and is a sequence of aryl rings linked with covalent bonds $[8,20]$. This assumption is confirmed by infrared spectroscopy $[8,20]$. At the same time, NMR data show that polydopamine aggregates consist of monomers crosslinked by non-covalent hydrogen bonds and $\pi-\pi$ interactions [21]. A possibility of self-assembly in solution and the subsequent polymerization with the formation of covalent bonds has also been considered [17]. It has been shown recently that all these mechanisms are not in contradiction [22]: the reactions compete with each other, and the conditions of polymerization such as $\mathrm{pH}$, oxidizer type and the concentration of dopamine hydrochloride determine the mechanism of polymerization.

Polydopamine films formed at the air-water interface have attracted less attention [16,23-26] as compared with the films on a solid substrate $[27,28]$. The application of pendant drop tensiometry showed that the surface tension decreased to $53 \mathrm{mN} / \mathrm{m}$ at a concentration of $2 \mathrm{~g} / \mathrm{l}$ and to $10 \mathrm{mN} / \mathrm{m}$ at a concentration of $10 \mathrm{~g} / 1$ [24]. At the same time, it follows from the images of the drop profile that the film pulls out the drop and its shape differs significantly from that described by the standard Young - Laplace equation. Besides, the polydopamine film is far more extended than a monolayer, and one can measure only some effective surface tension, which can depend on the measurement method [29,30]. Even so, such films are characterized by weak durability, which can be improved by the addition of different agents such as urea, pyrochatechol, alginate catechol [25], poly(ethylene imine) [31], and metal ions [26]. Polymers work like cross-linkers creating covalent bonds between isolated polydopamine clusters. The mechanical properties of films strongly depend on their structure.

The main aim of the present study is elucidation of the mechanism of the formation of a polydopamine film at the surface of aqueous solutions of dopamine hydrochloride. To this aim, three surface-sensitive experimental techniques were applied to the problem. First, dynamic dilatational surface rheology can be especially useful if the surface layer contains two-dimensional aggregates [29,32,33]. Second, ellipsometry can give information about the adsorption extent and kinetics of formation of surface films $[34,35]$. Lastly, Brewster angle microscopy can give information about the lateral heterogenties present in surface films [36,37].

\section{Experimental}

In this work the oscillating ring method was applied to measure the dynamic dilatational surface elasticity. A detailed description of the method is given elsewhere $[38,39]$. The oscillations of the surface area were caused by the periodical motion of a glass ring partly immersed into investigated solution and the induced oscillations of the surface tension were measured by the Wilhelmy plate method. In this case the 
oscillations of the surface tension and surface area can be represented in complex form. If the oscillation amplitudes are small enough, one can define the dynamic surface elasticity as a ratio of the complex surface tension increment $\Delta \gamma$ and relative changes of the surface area $\Delta A / A$.

The real and imaginary parts of the dynamic surface elasticity can be calculated from the amplitudes of the surface tension $|\Delta \gamma|$ and the relative surface area $|\triangle A / A|$, and also from the phase shift between the oscillations of these two quantities $\phi$ :

$$
E=E_{R}+i \cdot E_{i}=\left|\frac{A \Delta \gamma}{\Delta A}\right|(\cos \varphi+i \sin \varphi)
$$

The films of polydopamine are characterized by almost elastic behavior and the imaginary part of dynamic surface elasticity is much less than the real one.

Kinetic dependencies of the refractive index and surface layer thickness were estimated from ellipsometric angles using a null-ellipsometer Multiskop (Optrel GBR, Germany) operating with a single wavelength of $632.8 \mathrm{~nm}$. The angle of incidence was chosen close to the Brewster angle in order to provide high sensitivity to the properties of the polydopamine film. The change of the light polarization after reflection from the solution surface depends on the surface layer composition and can be characterized by two ellipsometric angles $(\Psi$ and $\Delta)$ :

$$
\tan \Psi e^{i \Delta}=\frac{r_{p}}{r_{s}}
$$

In this relation $r_{p}$ and $r_{s}$ are the complex reflection coefficients corresponding to the field components parallel and perpendicular to the reflection plane respectively.

In the case of a homogeneous film with a thickness much less than the wavelength ellipsometric angles can be connected with the thickness and refractive index of the film [40,41]. A numerical iteration procedure was applied with this aim.

The morphology of polydopamine at the air-water interface was determined by Brewster angle microscopy (BAM) using BAM 1 (Nanofilm Technology, Germany).

Dopamine hydrochloride (purity 98\%) was purchased from Sigma-Aldrich and used without further purification. All the solutions at $\mathrm{pH} 8.5$ were prepared in Trizma buffer (Sigma Aldrich) with an ionic strength of $0.01 \mathrm{M}$. Triply distilled water was used for these purposes. The surface tension of the buffer solutions was $72.8 \mathrm{mN} / \mathrm{m}$. The surface properties were measured at room temperature $\left(20 \pm 1^{\circ} \mathrm{C}\right)$.

\section{Results and discussion}

The dynamic properties of polydopamine films formed on the surface of aqueous solutions of dopamine hydrochloride were measured as a function of surface age and initial bulk concentration. The oscillation ring method, ellipsometry and BAM showed significant changes of the surface properties in the course of the polymerization process.

Only the surface tension measured at a constant surface area turned out to be insensitive to the process of polymer formation and was close to the value for pure water. The corresponding difference did not exceed $3 \mathrm{mN} / \mathrm{m}$. The Wilhelmy plate method was used to determine the surface tension values in both cases with and without deformations, and two different experimental setups were used for these purposes. Application of plates made from paper, glass and platinum led to the same results to within the error limits. Although the measured surface tension could differ from the literature data, it was quite reproducible. Measurements of the surface tension in previous studies of the same system were performed by the pendant drop method [16, 24]. This method allowed measurements at shorter surface ages and proved to be effective if the adsorption layer did not contain microparticles, was thin enough and not rigid. Some of these conditions are probably not fulfilled for thick polydopamine adsorption layers and the drop shape cannot be described by the static Laplace equation [16,24]. In this case, the commercial automatic instrument can keep the volume of the drop constant by pumping the solution inside the drop. The corresponding small oscillations of the drop area can influence the obtained results. 
In the case of the surface tension measured under surface area oscillations, the mean values (averaged over compression and expansion cycles) decreased with an increase of the initial bulk concentration of dopamine hydrochloride up to $1.5 \mathrm{~g} / 1$ while for more concentrated samples the values increased again (Fig. 1). Note that the experimental points in Fig. 1 represent averaged data over $4-5$ periods of ring oscillations. In the course of surface tension oscillations when the film expands, the surface tension approaches the value for pure solvent and remains approximately constant with further expansion up to a certain moment during compression, when it starts to decrease again (Fig. 2A). The kinetic dependencies of the dynamic surface tension at the maximum compression and expansion (Fig. 2B) show that values change with time only upon compression, while the surface tension is approximately constant with time upon expansion. If the oscillations are stopped at a certain position of the ring when the surface pressure deviates from zero, the system preserves this value during the observation time.

The rise in the reported surface tension for samples at the highest measured initial bulk concentrations of dopamine hydrochloride may be initially surprising, as this would not be expected for a system at thermal and chemical equilibrium. However, if the thickness of the adsorption layer increases significantly due to, for example, adsorption of micro- and nanoparticles or formation of multilayers, one can then measure only an 'effective surface tension' [29,42]. As opposed to the adsorption of a simple surfactant in dynamic adsorption/desorption equilibrium, the adsorption of particles or the formation of a relatively thick film (or some patches of multilayers) results in a change of the free energy of the interface that cannot be predicted by Gibb's equation [29,42]. As a result, the effective surface tension no longer represents the equilibrium surface tension of the system.

The mean surface tension of a sample of $1 \mathrm{~g} / \mathrm{l}$ dopamine hydrochloride decreased to about $66 \mathrm{mN} / \mathrm{m}$ in the course of surface area oscillations. This value is slightly higher than the data of Ponzio et al.[16], probably due to the difference in experimental methods.

It is noteworthy that significant changes of the surface tension of the investigated solutions were observed only in the case of surface area oscillations. Since the surface tension of dopamine hydrochloride solutions is close to the water value, the changes of this quantity (Fig. 1) can be attributed to the polydopamine formation in the surface layer as a result of a chemical reaction at the interface with oxygen from the air and not the polydopamine adsorption. Although the formation of polydopamine was observed at all concentrations studied, at low concentrations $(\leq 0.5 \mathrm{~g} / \mathrm{l})$, this phenomenon was observed only at long surface life times ( $>5$ hours; data not shown). Significant changes of the effective surface tension occur only at strong compression indicating that the surface layer consists of separate domains, which do not interact before the decrease of the distance between them to a certain value.

The surface properties of polydopamine formation at the air-water interface depended strongly on the initial concentration of dopamine hydrochloride, and the whole concentration range can be divided into three ranges of low $(\leq 0.5 \mathrm{~g} / 1)$, middle $(0.75-1.5 \mathrm{~g} / \mathrm{l})$ and high $(\geq 2 \mathrm{~g} / \mathrm{l})$ concentrations according to the characteristic features of the kinetic and concentration dependencies of surface properties. As such, the following description will be split into the samples from these three ranges.

In the low concentration range $(\leq 0.5 \mathrm{~g} / \mathrm{l})$, the dynamic surface elasticity (Fig.3) and the dynamic surface tension (Fig.1) are close to the values for pure water. At the same time, it is obvious that the formation of polydopamine takes place in the solution bulk. The solution color changes and becomes finally dark brown. Although changes of the surface properties were not detected by the oscillation ring method, other methods (BAM and ellipsometry) indicated some deviations from the behavior of pure water. At low concentrations BAM images showed only a continuous gray background like in the case of pure water for about $10 \mathrm{~h}$ (Fig.4A). The perturbation of the surface by a thin needle caused no effects. Only after the surface compression by about three times (Fig.4B) bright regions corresponding to the polydopamine domains could be observed. The ellipsometric signal fluctuated strongly at these concentrations (data not shown). These fluctuations exceed the error limits and indicated the macroheterogeneity of the layer and the mobility of polydopamine domains. Similar fluctuations have been noted previously for adsorbed layers of human serum albumin[37]. It is possible to assume that the some loose domains of polydopamine are formed at the interface at low concentrations. They do not form a continuous layer and their number is not enough to change the dynamic surface elasticity and effective surface tension.

In the middle concentration range $(0.75-1.5 \mathrm{~g} / \mathrm{l})$, the dynamic surface elasticity noticeably increased while the effective dynamic surface tension decreased. The dynamic surface elasticity reaches about 30 $\mathrm{mN} / \mathrm{mat}$ a concentration of $0.75 \mathrm{~g} / \mathrm{l}$ (Fig.3). Increase of the concentration to $1 \mathrm{~g} / \mathrm{l}$ and $1.5 \mathrm{~g} / \mathrm{l}$ leads to an 
increase of the dynamic surface elasticity to about $60 \mathrm{mN} / \mathrm{m}$ (Fig.3). This value is comparable with those for adsorption layers of globular proteins or monolayers of soft nanoparticles [29] and indicates strong interactions of polydopamine domains in the surface layer. The effective surface tension in the middle concentration range has the lowest values (about $66 \mathrm{mN} / \mathrm{m}$ ) of all the investigated concentrations (Fig. 1). Fig. 2A and 2B show that the surface tension decreases only during surface compression. This observation may indicate that a continuous polymer film is not formed for these samples in the middle concentration range. The number of polydopamine domains or their size could increase during the surface film formation but they are still not interconnected or the contacts between them are broken under deformations during the time of observations.

Fig. 5 shows the evolution of a polydopamine film at an initial bulk concentration of $1 \mathrm{~g} / \mathrm{l}$ using BAM. The surface is homogeneous at the start of the measurement (Fig.5A), but in about 30 minutes some heterogeneity appears (Fig. 5B). The patches of polydopamine look connected but there is still empty space between them. In this case the surface elasticity is about $10 \mathrm{mN} / \mathrm{m}$. The obtained images support the idea that the film consists of separate surface particles. Even at longer times, the surface layer is inhomogeneous (Fig.5C,D). Fig.5C shows that there is still empty surface between the domains. Although they are interconnected, the contacts between them are weak and can be broken under surface expansion. The particles do not merge and the repulsion between them results in a surface elasticity of about $60 \mathrm{mN} / \mathrm{m}$. In 10 hours (Fig. 5D) the packing of the surface particles becomes tighter.

As a result of measurement of the kinetic dependencies of the ellipsometric angles $\Delta$ and $\psi$, it was possible to calculate kinetic dependencies of the film refractive index (Fig. 6A) and film thickness (Fig. 6B). The fluctuations of ellipsometric data are also observed in the middle concentration range. In about 2-3 hours after surface formation, the fluctuations disappear (Fig.6A,B). This time almost coincides with the formation time of an immobile layer of polydopamine domains (surface particles) observed by BAM, and complements the previous results from human serum albumin when ellipsometric fluctuations disappeared as films grew to have higher surface coverage [37]. The fluctuations of the signal decrease strongly at longer times. The obtained results show that the refractive index of a pure polydopamine layer $(1.73 \pm 0.02 \mathrm{i}[16,19])$ is not reached in the middle concentration range. The refractive index for $1 \mathrm{~g} / \mathrm{l}$ solution starts from the value corresponding to pure water and increases up to 1.66 during 16 hours. Probably this is a consequence of the hydration of polydopamine in the adsorption layer and the layer heterogeneity. The kinetic dependencies of the film thickness, unlike the dependencies of the refractive index, display two main steps of the surface film formation. The first short step corresponds to the very fast changes of the film thickness and can be associated with the formation of primary domains in the surface layer with a thickness of about $40 \mathrm{~nm}$. During the second longer step the domains are almost immobile and the thickness grows slower. These observations are consistent with the possibility that the surface layer contains only molecules of dopamine hydrochloride just after the surface formation (Fig. 7A). After that the first domains of polydopamine are formed at the air-water interface because of the oxidation of dopamine hydrochloride by oxygen from the air (Fig. 7B). These domains are very mobile and move along the surface leading to strong fluctuations of the ellipsometric signal. The size and/or the number of the patches increase fast during the first hour after the surface formation (Fig. 7C). After that the refractive index continues to increase smoothly while the thickness of the film starts to increase slowly when the polymer fills the gaps between the domains (Fig. 7D). It follows that oxidation of dopamine hydrochloride by oxygen in direct contact with air is faster than the corresponding oxidation by oxygen dissolved in water. Therefore the domains grow mainly in the directions parallel to the air-water interface.

In the high concentration range ( $\geq 2 \mathrm{~g} / 1)$, the surface properties display a distinct type of behavior. The results of the oscillation ring method in this concentration range differ significantly from those of the lower and middle concentrations (Figs. 1 and 3). For surface ages of less than 2 hours, the surface tension and the surface elasticity change only slightly after that (Fig. 3). Indeed the dynamic surface elasticity does not exceed $10 \mathrm{mN} / \mathrm{m}$ even at longer surface lifetimes ( $>3$ hours). The results may be explained by the fact that polymerization in this case is faster and one can observe the behavior specific for a continuous homogeneous and fragile surface film even at short film lifetimes. If the surface age is longer than one hour, the adsorption layer does not contain separate polydopamine domains but the surface area oscillations lead to the destruction of the uniform polymer film. It can fold under compression or break under expansion into pieces with sizes larger than the size of primary domains. These pieces can overlap and form multilayers. In 
this case the dynamic surface elasticity is relatively low but the switching off the oscillations for a few hours leads to a very high surface elasticity of about $150 \mathrm{mN} / \mathrm{m}$, which decreases fast to about $10 \mathrm{mN} / \mathrm{m}$ again as a result of surface oscillations. The application of complementary techniques is needed to corroborate the physical picture of the film growth in this system.

The BAM images in this third concentration range also differ from those recorded at the lower and middle concentrations. Fig. 8 shows that there is significantly different behavior in how the morphology of a polydopamine film at an initial bulk dopamine hydrochloride concentration of $2 \mathrm{~g} / \mathrm{l}$ changes with time. At the beginning, one can observe only a gray background corresponding to pure water (Fig. 8A). In 30 minutes some large aggregates become visible (Fig.8B), but they disappear during next 15 minutes (Fig. 8C) and the film looks homogeneous, bearing in mind the spatial resolution of the measurement of a couple of micrometers. In one hour (Fig. 8D) the image is similar to that for lower concentrations and higher surface ages (Fig.5C). After that the surface becomes gradually more homogeneous and in 100 minutes one can see again a uniform grey background (Fig. 8E) as it was observed at the beginning of the layer formation. The issue is then raised if the layer has disappeared or a layer remains but with any remaining lateral features reduced below the spatial resolution of the technique. One way to distinguish the formation of a continuous layer as opposed to no layer is through application of a weak mechanical disturbance with a thin needle leading to the destruction of the continuous layer (Fig. 8F). In this case one can observe some bright regions corresponding to the polydopamine layer and dark regions of the water surface. The obtained results not only confirm the formation of a uniform homogeneous film but also show that the same steps of the layer formation which were observed for the middle concentrations are prevalent in this case. At a concentration of $5 \mathrm{~g} / \mathrm{l}$, similar results were obtained, but the formation of two-dimensional domains was not observed during the surface polymerization, which can be attributed to the higher polymerization rate.

The ellipsometric data show a noticeable difference between the ranges of the high and middle concentrations (Fig. 6A,B). There is also an important difference between the two studied high concentrations of 2 and $5 \mathrm{~g} / \mathrm{l}$ (Fig 6B). The refractive index at a concentration of $5 \mathrm{~g} / \mathrm{l}$ increases monotonically for 16 hours after the surface formation approaching 1.706, which is close to the value for a pure polydopamine film. The corresponding maximum value at the concentration of $2 \mathrm{~g} / \mathrm{l}$ solution is only 1.662 , probably due to the contribution of incomplete coverage of the polydopamine layer that could not be detected below the spatial resolution of the BAM. At these concentrations one can observe the same fluctuations of the ellipsometric signal at the beginning of the layer formation as in the case of lower concentrations but for shorter times. The differences between the results for $2 \mathrm{~g} / \mathrm{l}$ and $5 \mathrm{~g} / \mathrm{l}$ solutions can be attributed to the difference in polymerization rates. Analysis of the data for samples at these high concentrations leads to an additional step of the adsorption layer formation as compared with the case of the middle concentrations.

It is possible to display a scheme of the mechanism of layer formation in the high concentration range as follows: formation of initial domains (Fig. 7A,B), their growth (Fig. 7C,D) and filling the gaps between the domains (Fig. 7E,F) before subsequent film growth in the direction normal to the surface (Fig. 7G,H). It can be inferred that the last step is caused by the oxidation of dopamine hydrochloride by oxygen dissolved in water after formation of a dense polydopamine film in the proximal region of surface layer. The film reduces access of oxygen from the air to the dissolved dopamine hydrochloride and the process is slower than the initial growth of primary domains in contact with air. The last step at a concentration of $2 \mathrm{~g} / \mathrm{l} \mathrm{starts}$ only at longer times and one can see significant changes of thickness become evident only in 15 hours after the surface formation.

Fig. 9 shows a scheme displaying different steps of polydopamine film formation at different initial bulk concentrations of dopamine hydrochloride. At the first stage of polymerization, the layer consists of separate domains at the air-water interface. The domains become connected gradually and after a certain time the film become homogeneous. It is possible to distinguish between three main types of the adsorption layer structure leading to some differences in the kinetic dependencies of the dynamic surface elasticity and dynamic surface tension. At low initial bulk concentrations of dopamine hydrochloride $(\leq 0.5 \mathrm{~g} / \mathrm{l})$, the number of domains is small and they do not interact. In this case the dynamic surface elasticity is zero (Fig.9A). In the middle concentration range (0.75-1.5 g/l), the domains grow and become connected. When the film is expanded the links between the domains break and the surface properties are close to those for pure water (Fig. 9B). This state corresponds to a constancy of the surface tension at the pure water value in 
the course of expansion of the oscillations (Fig.2A). Film compression leads to interactions between relatively rigid domains and one can observe a decrease of the surface tension and an increase of the surface elasticity. In this case the polydopamine domains behave like nanoparticles in a monolayer. After that the surface area between the domains become gradually filled with polydopamine. In this case the surface deformation leads to the destruction of the links between the domains. Without deformations or at higher concentrations these primary domains form a continuous homogeneous film. At high concentrations $(\geq 2 \mathrm{~g} / \mathrm{l})$, the polymerization is faster and a dense almost continuous two-dimensional film is formed already for about two hours (Fig. 9C). At longer surface life times the film becomes denser and thicker. This process leads to a homogeneous and loose surface film with a relatively low surface elasticity. Compression of this film can lead to its folding while expansion leads to its breaking.

\section{Conclusions}

The oxidation of dopamine hydrochloride solutions results in the formation of surface films that have a very interesting interfacial morphology affected by competing chemical processes in the bulk and at the air-water interface. The kinetic dependencies of the dynamic surface elasticity, film thickness and refractive index, and the lateral film heterogeneity have allowed in this work determination of the main steps of polydopamine layer formation with respect to the initial bulk concentration of the reactant. The dynamic surface elasticity proved to be sensitive to the transition from separate domains to a continuous twodimensional polymer film. Some patches of polydopamine at the interface become centers for further polymerization. The formation of relatively rigid polymer domains in the surface layer and their interactions result in an increase of the dynamic surface elasticity in the course of the layer formation. In this case, in effect, the polydopamine domains act as nanoparticles embedded in the surface monolayer. For all samples except the highest bulk initial concentrations of dopamine hydrochloride, the surface film is not entirely homogeneous even at the longest surface lifetimes. The data for the high concentration samples, in contrast, show a transition from a state of close packed separate domains, which are also observed for lower concentrations at long surface lifetimes, to a homogeneous uniform film. These films have low surface elasticity as a result of the loss of their integrity during deformation.

Overall, the results presented in this work have highlighted the complicated mechanism of film formation at the air-water interface involving the coalescence of aggregates during a surface chemical reaction in comparison with a relatively slow oxidation process in the bulk. The interpretation of concentration-dependent data during such a polymerization process has allowed elucidation of various aspects of surface film formation that have not been resolved to date. The application of several different surface-sensitive experimental techniques was key to the formation of this new interfacial picture.

\section{Acknowledgements}

This work was supported by the Russian Science Foundation (project no. 18-73-00108). The authors are also grateful to the "Geomodel" Research Center of Saint-Petersburg State University.

\section{References}

[1] Y. Liu, K. Ai, L. Lu, Polydopamine and its derivative materials: Synthesis and promising applications in energy, environmental, and biochemical fields, Chem. Rev. 114 (2014) 5057-5115. doi: $10.1021 / \mathrm{cr} 400407 \mathrm{a}$.

[2] J.H. Ryu, P.B. Messersmith, H. Lee, Polydopamine Surface Chemistry : A Decade of Discovery, (2018). doi:10.1021/acsami.7b19865.

[3] V. Ball, Polydopamine films and particles with catalytic activity, Catal. Today. 301 (2018) 196-203. doi:10.1016/j.cattod.2017.01.031.

[4] Y. Zheng, L. Zhang, J. Shi, Y. Liang, X. Wang, Z. Jiang, Mussel-inspired surface capping and pore filling to confer mesoporous silica with high loading and enhanced stability of enzyme, Microporous Mesoporous Mater. 152 (2012) 122-127. doi:10.1016/j.micromeso.2011.11.049. 
[5] H. Long, D. Del Frari, A. Martin, J. Didierjean, V. Ball, M. Michel, H.I. El Ahrach, Polydopamine as a promising candidate for the design of high performance and corrosion-tolerant polymer electrolyte fuel cell electrodes, J. Power Sources. 307 (2016) 569-577. doi:10.1016/j.jpowsour.2015.12.138.

[6] N. Zhang, W. Ma, P.G. He, Y.T. Long, A polydopamine derivative monolayer on gold electrode for electrochemical catalysis of H2O2, J. Electroanal. Chem. 739 (2015) 197-201.

doi:10.1016/j.jelechem.2014.12.007.

[7] N. Farnad, K. Farhadi, N.H. Voelcker, Polydopamine nanoparticles as a new and highly selective biosorbent for the removal of copper (II) ions from aqueous solutions, Water. Air. Soil Pollut. 223 (2012) 3535-3544. doi:10.1007/s11270-012-1131-7.

[8] F. Yu, S. Chen, Y. Chen, H. Li, L. Yang, Y. Chen, Y. Yin, Experimental and theoretical analysis of polymerization reaction process on the polydopamine membranes and its corrosion protection properties for 304 Stainless Steel, J. Mol. Struct. 982 (2010) 152-161.

doi:10.1016/j.molstruc.2010.08.021.

[9] R. Subair, B.P. Tripathi, P. Formanek, F. Simon, P. Uhlmann, M. Stamm, Polydopamine modified membranes with in situ synthesized gold nanoparticles for catalytic and environmental applications, Chem. Eng. J. 295 (2016) 358-369. doi:10.1016/j.cej.2016.02.105.

[10] A. Postma, Y. Yan, Y. Wang, A.N. Zelikin, E. Tjipto, F. Caruso, Self-polymerization of dopamine as a versatile and robust technique to prepare polymer capsules, Chem. Mater. 21 (2009) 3042-3044. doi:10.1021/cm901293e.

[11] M.E. Lynge, R. van der Westen, A. Postma, B. Städler, Polydopamine — a nature-inspired polymer coating for biomedical science, Nanoscale. 3 (2011) 4916. doi:10.1039/c1nr10969c.

[12] F. Ponzio, V. Ball, Persistence of dopamine and small oxidation products thereof in oxygenated dopamine solutions and in "polydopamine" films, Colloids Surfaces A Physicochem. Eng. Asp. 443 (2014) 540-543. doi:10.1016/j.colsurfa.2013.12.027.

[13] H.-C. Yang, Q.-Y. Wu, L.-S. Wan, Z.-K. Xu, Polydopamine gradients by oxygen diffusion controlled autoxidation, Chem. Commun. 49 (2013) 10522. doi:10.1039/c3cc46127k.

[14] W. Zheng, H. Fan, L. Wang, Z. Jin, Oxidative Self-Polymerization of Dopamine in an Acidic Environment, Langmuir. 31 (2015) 11671-11677. doi:10.1021/acs.langmuir.5b02757.

[15] L. Shang, L. Yang, J. Seiter, M. Heinle, G. Brenner-Weiss, D. Gerthsen, G.U. Nienhaus, Nanoparticles Interacting with Proteins and Cells: A Systematic Study of Protein Surface Charge Effects, Adv. Mater. Interfaces. 1 (2014) 1300079. doi:10.1002/admi.201300079.

[16] F. Ponzio, P. Payamyar, A. Schneider, M. Winterhalter, J. Bour, F. Addiego, M.P. Krafft, J. Hemmerle, V. Ball, Polydopamine films from the forgotten Air/water interface, J. Phys. Chem. Lett. 5 (2014) 3436-3440. doi:10.1021/jz501842r.

[17] S. Hong, Y.S. Na, S. Choi, I.T. Song, W.Y. Kim, H. Lee, Non-covalent self-assembly and covalent polymerization co-contribute to polydopamine formation, Adv. Funct. Mater. 22 (2012) 4711-4717. doi:10.1002/adfm.201201156.

[18] V. Ball, D. Del Frari, M. Michel, M.J. Buehler, V. Toniazzo, M.K. Singh, J. Gracio, D. Ruch, Deposition Mechanism and Properties of Thin Polydopamine Films for High Added Value Applications in Surface Science at the Nanoscale, Bionanoscience. 2 (2012) 16-34. doi:10.1007/s12668-011-0032-3.

[19] F. Bernsmann, V. Ball, A. Ponche, M. Michel, D.A. Gracio, D. Ruch, Dopamine - Melanin Film Deposition Depends on the Used Oxidant and Buffer Solution, Langmuir. 27 (2011) 2819-2825.

[20] R.A. Zangmeister, T.A. Morris, M.J. Tarlov, Characterization of polydopamine thin films deposited at short times by autoxidation of dopamine, Langmuir. 29 (2013) 8619-8628. doi:10.1021/la400587j.

[21] D.R. Dreyer, D.J. Miller, B.D. Freeman, D.R. Paul, C.W. Bielawski, Elucidating the Structure of Poly(dopamine), Langmuir. 28 (2012) 6428-6435. doi:10.1021/la204831b. 
[22] N.F. Della Vecchia, R. Avolio, M. Alfè, M.E. Errico, A. Napolitano, M. D’Ischia, Building-block diversity in polydopamine underpins a multifunctional eumelanin-type platform tunable through a quinone control point, Adv. Funct. Mater. 23 (2013) 1331-1340. doi:10.1002/adfm.201202127.

[23] F. Ponzio, V. Ball, Polydopamine deposition at fluid interfaces, Polym Int. 65 (2016) 1251-1257. doi:10.1002/pi.5124.

[24] H. Abe, T. Matsue, H. Yabu, Reversible Shape Transformation of Ultrathin Polydopamine- Stabilized Droplet, Langmuir. 33 (2017) 6404-6409. doi:10.1021/acs.langmuir.7b01355.

[25] F. Ponzio, V. Le Houerou, S. Zafeiratos, C. Gauthier, T. Garnier, L. Jierry, V. Ball, Robust AlginateCatechol@Polydopamine Free-Standing Membranes Obtained from the Water/Air Interface, Langmuir. 33 (2017) 2420-2426. doi:10.1021/acs.langmuir.6b04435.

[26] X. Han, F. Tang, Z. Jin, Free-standing polydopamine films generated in the presence of different metallic ions: the comparison of reaction process and film properties, RSC Adv. 8 (2018) 1834718354. doi:10.1039/c8ra02930j.

[27] S.C. Goh, Y. Luan, X. Wang, H. Du, C. Chau, H.E. Schellhorn, J.L. Brash, H. Chen, Q. Fang, Polydopamine-polyethylene glycol-albumin antifouling coatings on multiple substrates, J. Mater. Chem. B. 6 (2018) 940-949. doi:10.1039/C7TB02636F.

[28] Q. Wei, F. Zhang, J. Li, B. Li, C. Zhao, Oxidant-induced dopamine polymerization for multifunctional coatings, Polym. Chem. 1 (2010) 1430. doi:10.1039/c0py00215a.

[29] B.A. Noskov, A.G. Bykov, Dilational rheology of monolayers of nano- and micropaticles at the liquid-fluid interfaces, Curr. Opin. Colloid Interface Sci. 37 (2018) 1-12. doi:10.1016/j.cocis.2018.05.001.

[30] J.W. Tavacoli, G. Katgert, E.G. Kim, M.E. Cates, P.S. Clegg, Size limit for particle-stabilized emulsion droplets under gravity, Phys. Rev. Lett. 108 (2012) 1-5. doi:10.1103/PhysRevLett.108.268306.

[31] H. Yang, W. Xu, Y. Du, J. Wu, Z. Xu, Composite free-standing films of polydopamine/ polyethyleneimine grown at the air/water interface, RSC Adv. 4 (2014) 45415-45418. doi:10.1039/c4ra04549a.

[32] B.A. Noskov, Protein conformational transitions at the liquid-gas interface as studied by dilational surface rheology, Adv. Colloid Interface Sci. 206 (2014) 222-238. doi:10.1016/j.cis.2013.10.024.

[33] B.A. Noskov, K.A. Timoshen, A. V Akentiev, N.S. Chirkov, I.M. Dubovsky, V.T. Lebedev, S.-Y. Lin, G. Loglio, R. Miller, V.P. Sedov, A.A. Borisenkova, Dynamic Surface Properties of Fullerenol Solutions, Langmuir. 35 (2019) 3773-3779. doi:10.1021/acs.langmuir.8b04152.

[34] A. Maestro, H.M. Hilles, F. Ortega, G. Rubio, F. Monroy, Reptation in langmuir polymer monolayers, Soft Matter. 6 (2010) 4407-4412. doi:10.1039/c0sm00250j.

[35] A. Tummino, J. Toscano, F. Sebastiani, B.A. Noskov, I. Varga, R.A. Campbell, Effects of Aggregate Charge and Subphase Ionic Strength on the Properties of Spread Polyelectrolyte/Surfactant Films at the Air/Water Interface under Static and Dynamic Conditions, Langmuir. 34 (2018) 2312-2323. doi:10.1021/acs.langmuir.7b03960.

[36] B. Li, Y. Wu, M. Liu, A.R. Esker, Brewster Angle Microscopy Study of Poly ( E -caprolactone ) Crystal Growth in Langmuir Films at the Air / Water Interface, Langmuir. 22 (2006) 4902-4905. doi: $10.1021 / \mathrm{la} 060048 \mathrm{~b}$.

[37] R.A. Campbell, J.C. Ang, F. Sebastiani, A. Tummino, J.W. White, Spread Films of Human Serum Albumin at the Air-Water Interface: Optimization, Morphology, and Durability, Langmuir. 31 (2015) 13535-13542. doi:10.1021/acs.langmuir.5b03349.

[38] J.J. Kokelaar, A. Prins, M. De Gee, A new method for measuring the surface dilational modulus of a liquid, J. Colloid Interface Sci. 146 (1991) 507-511. doi:10.1016/0021-9797(91)90214-S. 
[39] A.G. Bykov, S.-Y. Lin, G. Loglio, R. Miller, B. a. Noskov, Kinetics of Adsorption Layer Formation in Solutions of Polyacid/Surfactant Complexes, J. Phys. Chem. C. 113 (2009) 5664-5671. doi:10.1021/jp810471y.

[40] D. Ducharme, A. Tessier, R.M. Leblanc, Null ellipsometer for the studies of thin films at gas-water interface, Rev. Sci. Instrum. 58 (1987) 571-578. doi:10.1063/1.1139271.

[41] H. Motschmann, R. Teppner, Novel methods to study interfacial layers., Elsevier, 2001. doi:10.1080/10826068.2014.940969.

[42] R. Miller, V.B. Fainerman, V.I. Kovalchuk, D.O. Grigoriev, M.E. Leser, M. Michel, Composite interfacial layers containing micro-size and nano-size particles, Adv. Colloid Interface Sci. 128-130 (2006) 17-26. doi:10.1016/j.cis.2006.11.004. 


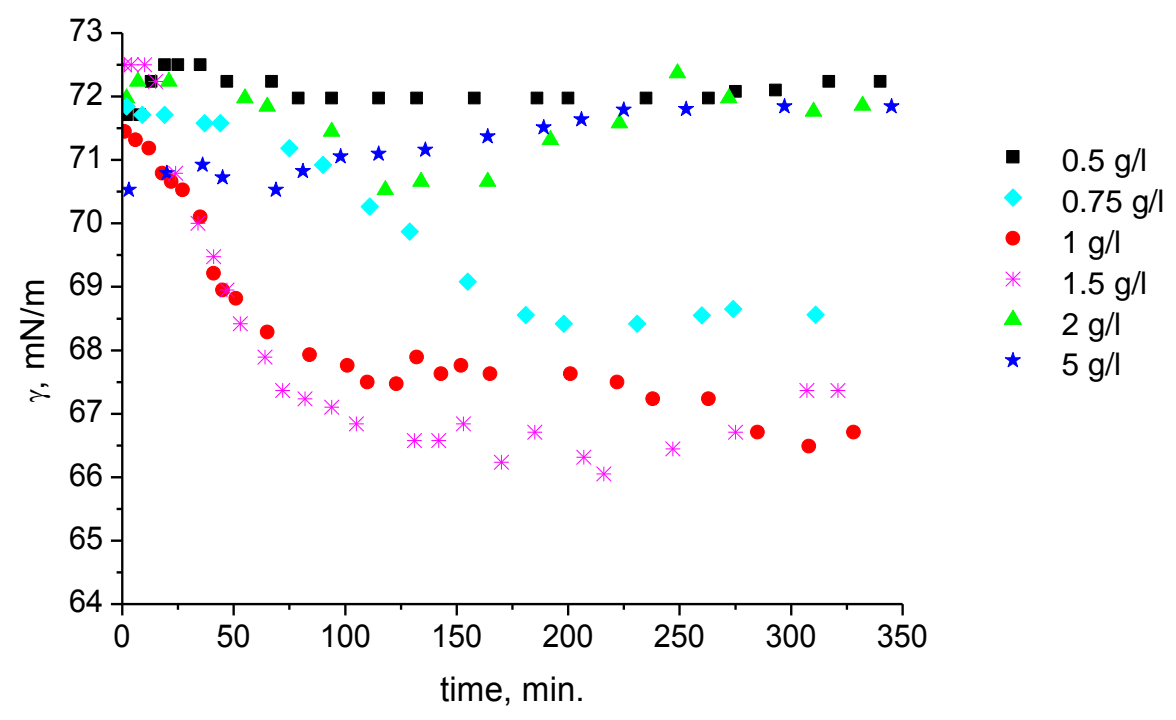

Fig. 1.Kinetic dependencies of the surface tension of dopamine hydrochloride solutions at $\mathrm{pH} 8.5$ and various initial bulk concentrations.
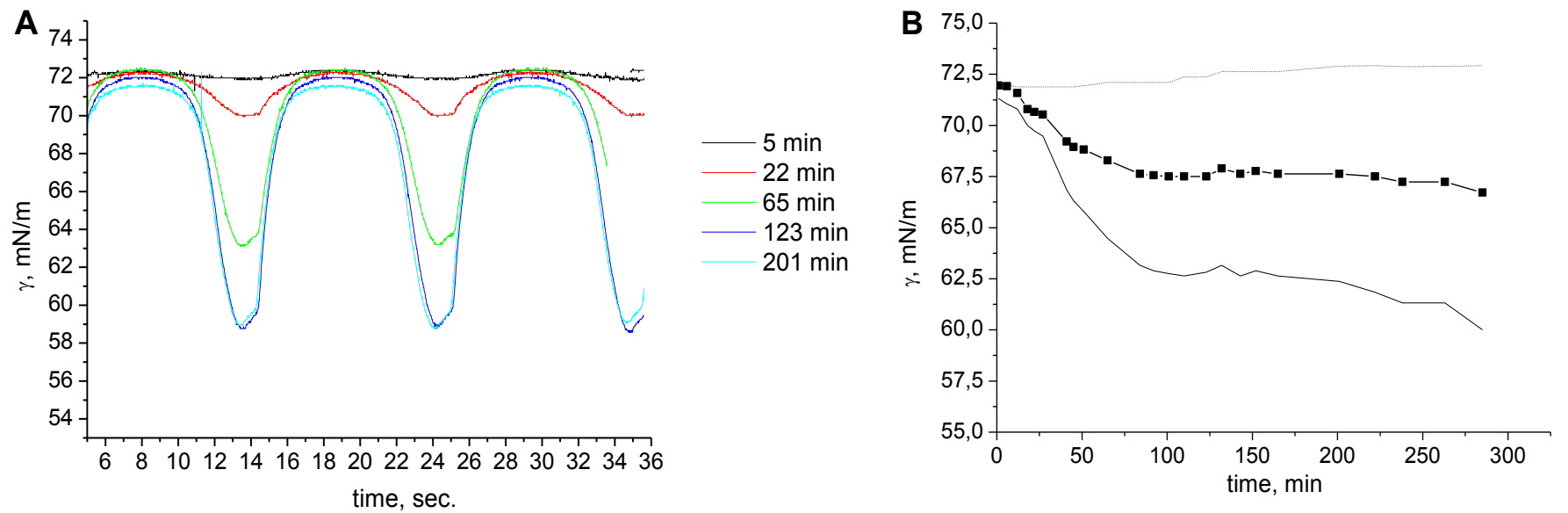

Fig. 2. A: Surface tension oscillations for dopamine hydrochloride solutions at $\mathrm{pH} 8.5$ and initial concentration $1 \mathrm{~g} / \mathrm{l}$ at an oscillation frequency of $0.1 \mathrm{~Hz}$ and amplitude of the surface area oscillations $0.7 \%$.

B: Kinetic dependencies of the surface tension of dopamine hydrochloride solutions at $\mathrm{pH} 8.5$ and initial concentration of $1 \mathrm{~g} / \mathrm{l}$. The solid line corresponds to the limiting values of the dynamic surface tension under compression and the dotted line under expansion 


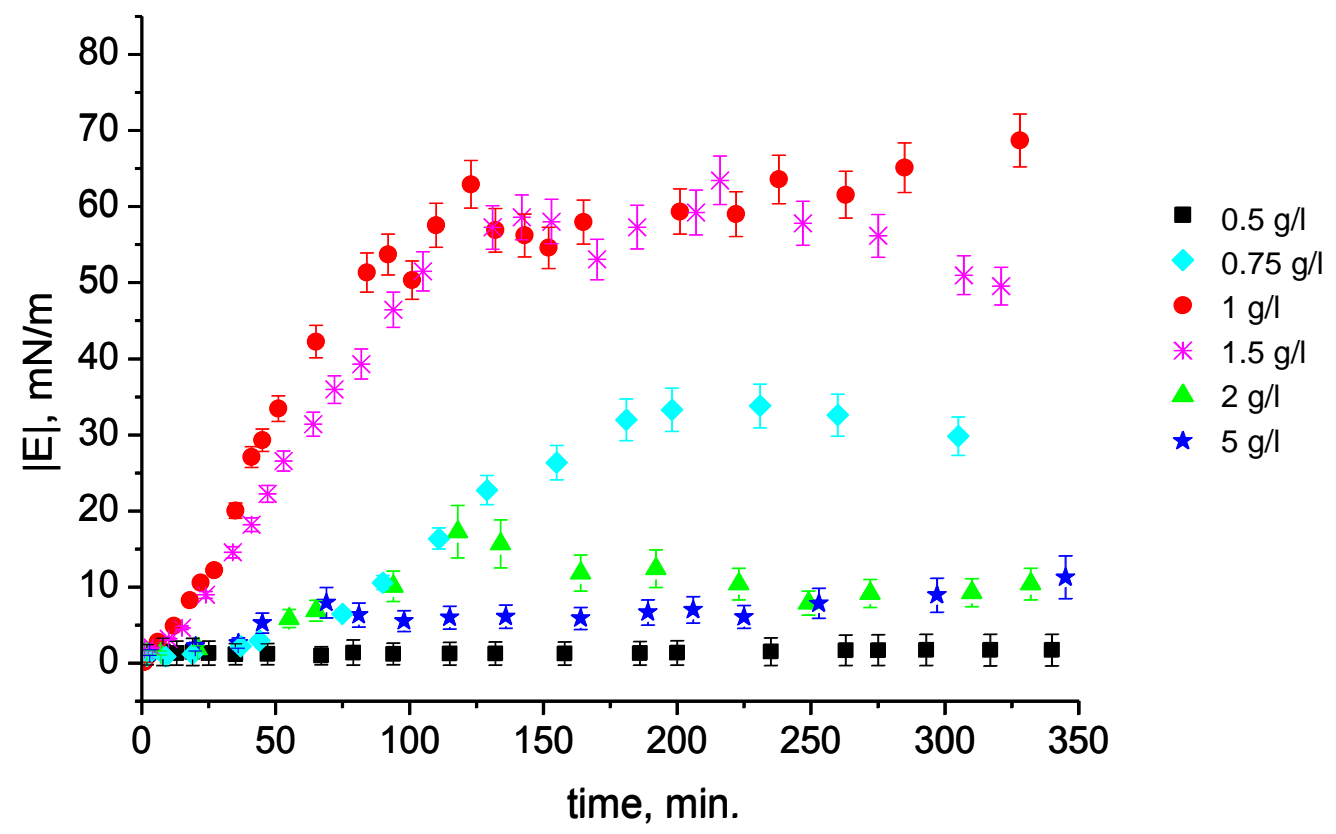

Fig. 3.Kinetic dependencies of the dynamic surface elasticity of dopamine hydrochloride solutions at $\mathrm{pH} 8.5$ and various initial concentrations.

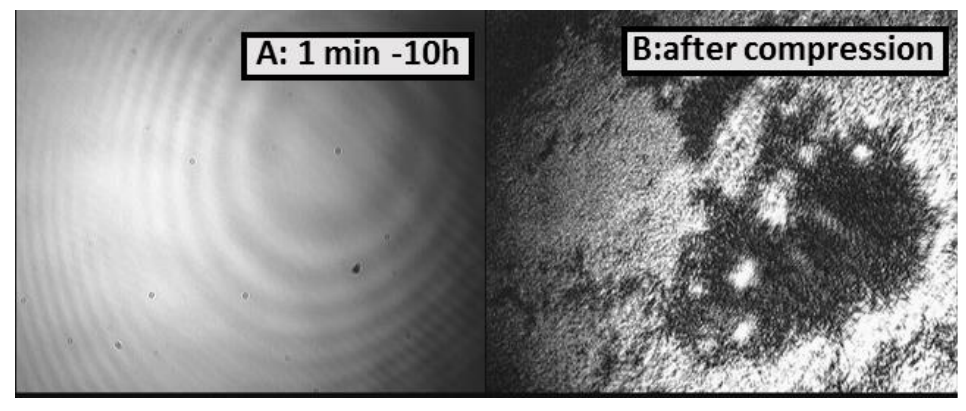

Fig.4.BAM images of polydopamine film formation from $0.5 \mathrm{~g} / 1$ dopamine hydrochloride solution at $\mathrm{pH} 8.5$. 


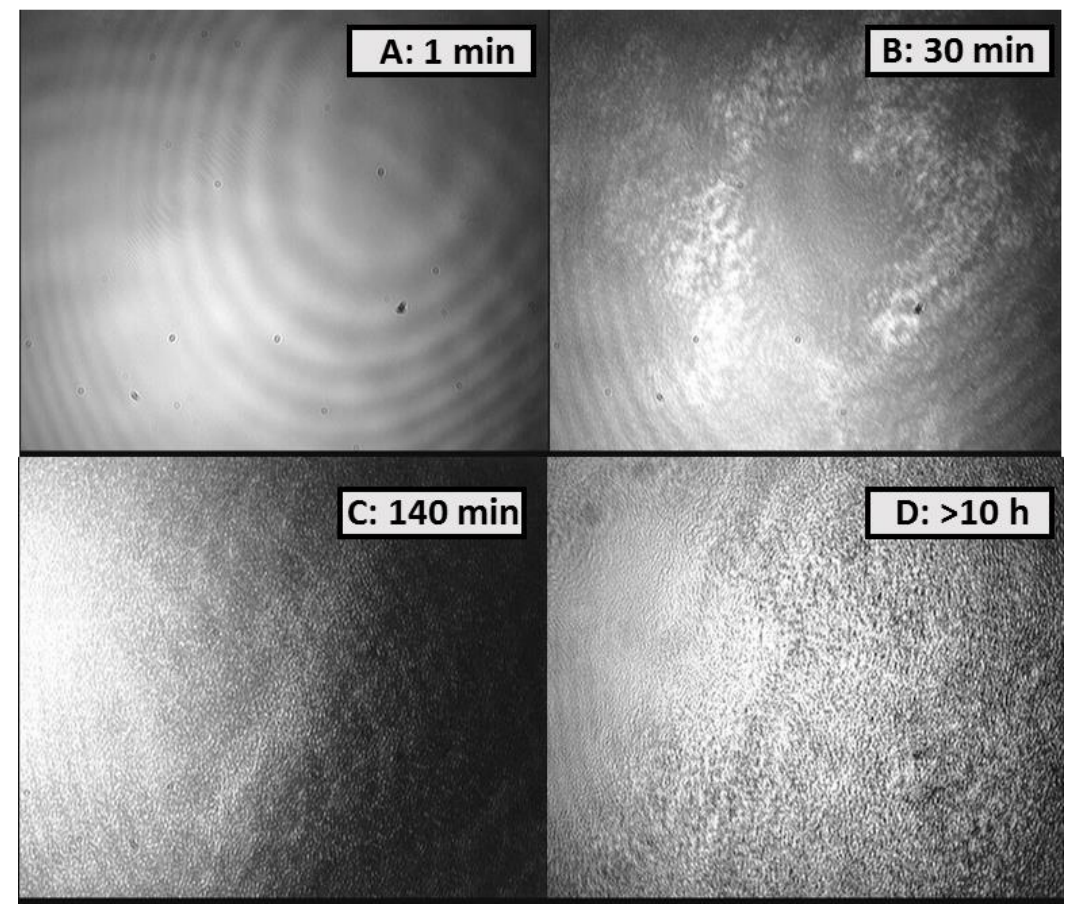

Fig.5. BAM images of polydopamine film formation from $1 \mathrm{~g} / \mathrm{l}$ dopamine hydrochloride solution at $\mathrm{pH}$ 8.5.
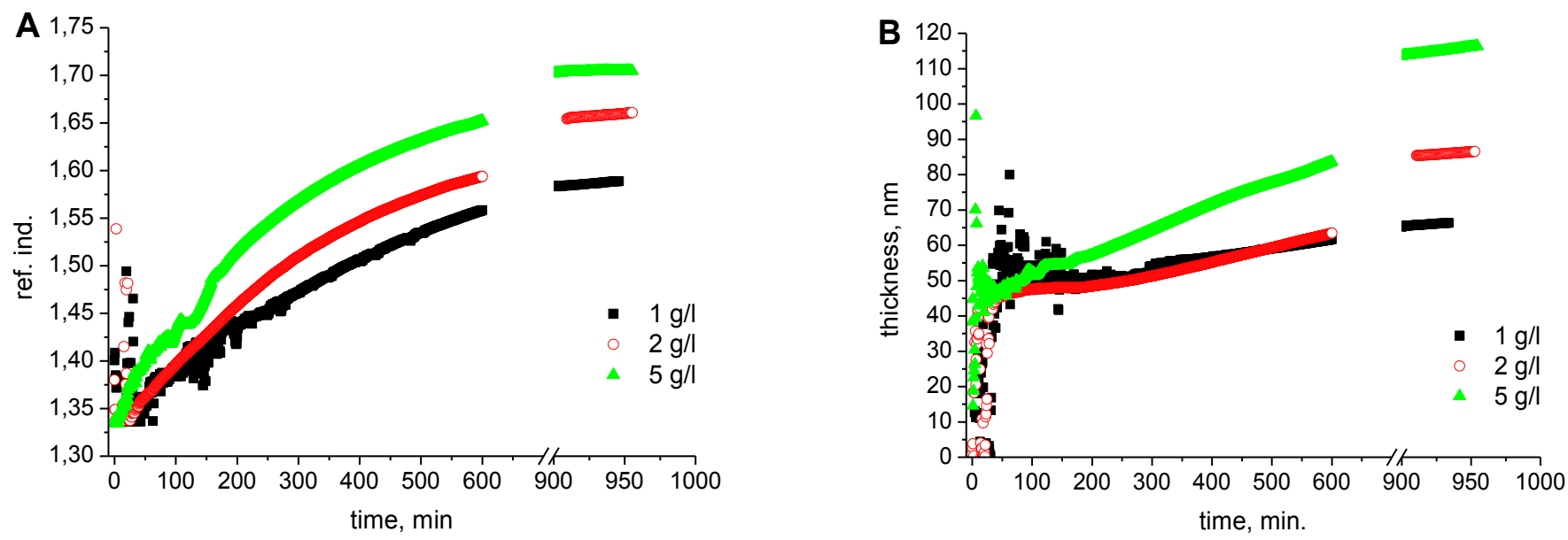

Fig.6.Kinetic dependencies of the film refractive index (A) and thickness (B) formed from dopamine hydrochloride solutions at $\mathrm{pH} 8.5$ and various initial bulk concentrations. 


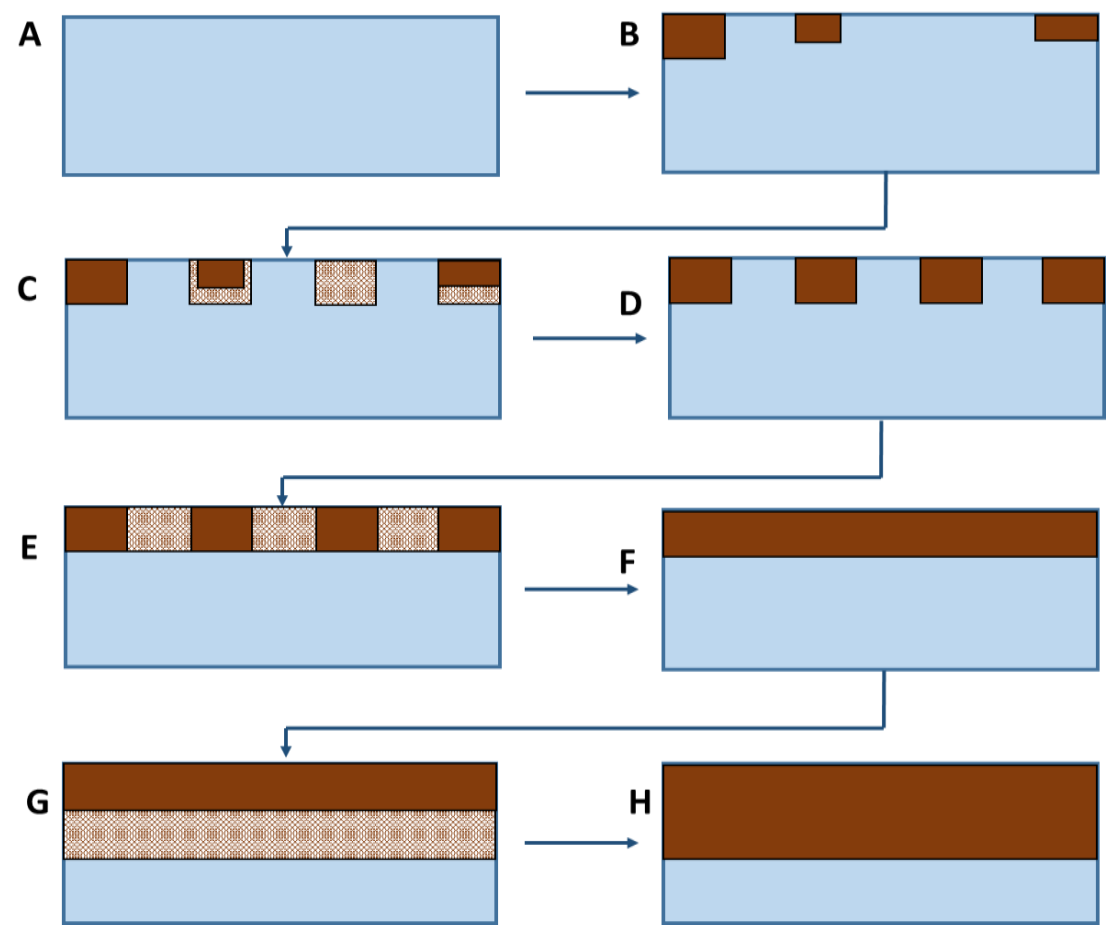

Fig. 7.A scheme of the change of polydopamine film morphology and thickness based on the data presented. The brown areas correspond to polydopamine already formed and the light-brown area corresponds to the directions of film growth.

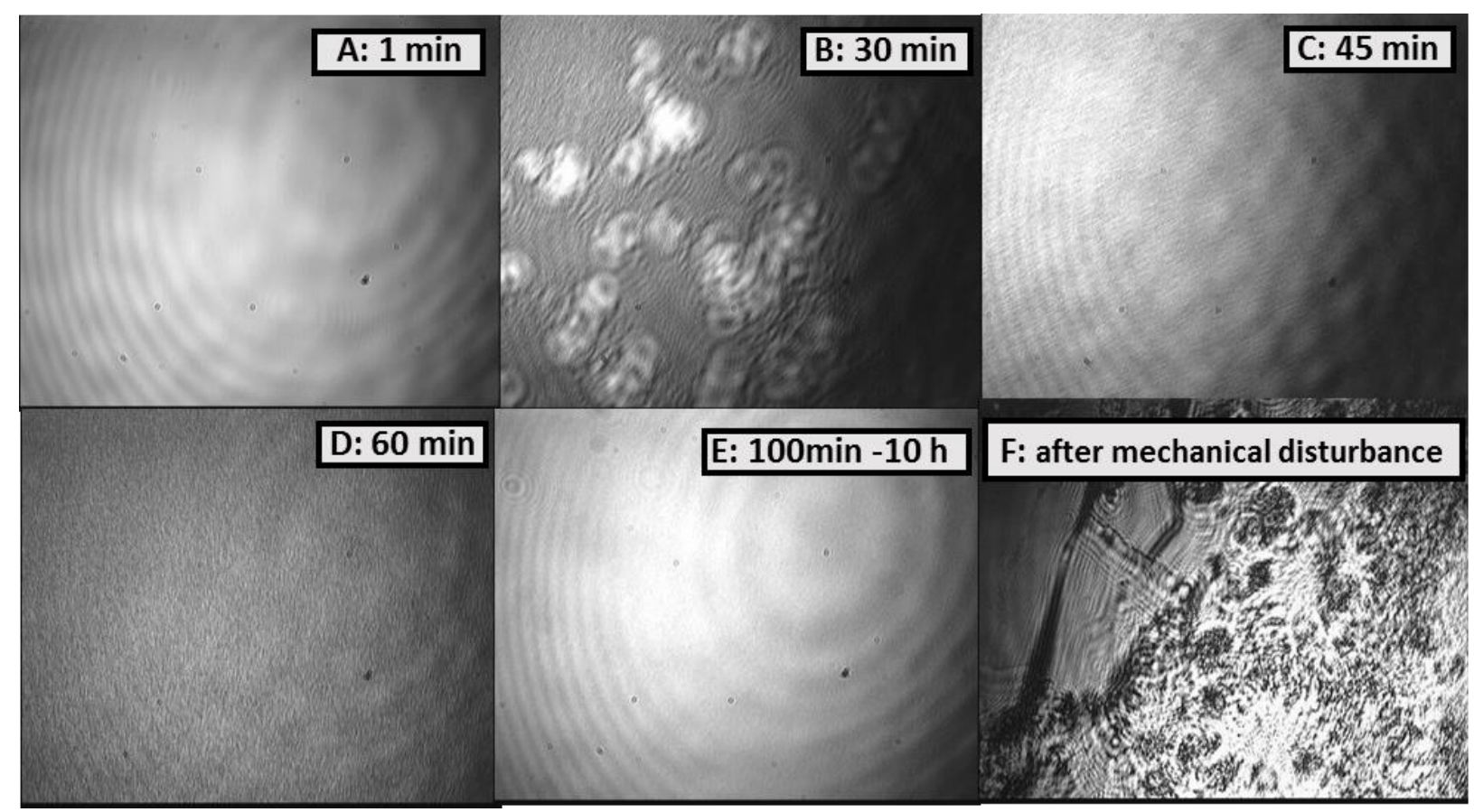

Fig.8. BAM images of polydopamine film formation from $2 \mathrm{~g} / \mathrm{l}$ dopamine hydrochloride solution at $\mathrm{pH}$ 8.5. 

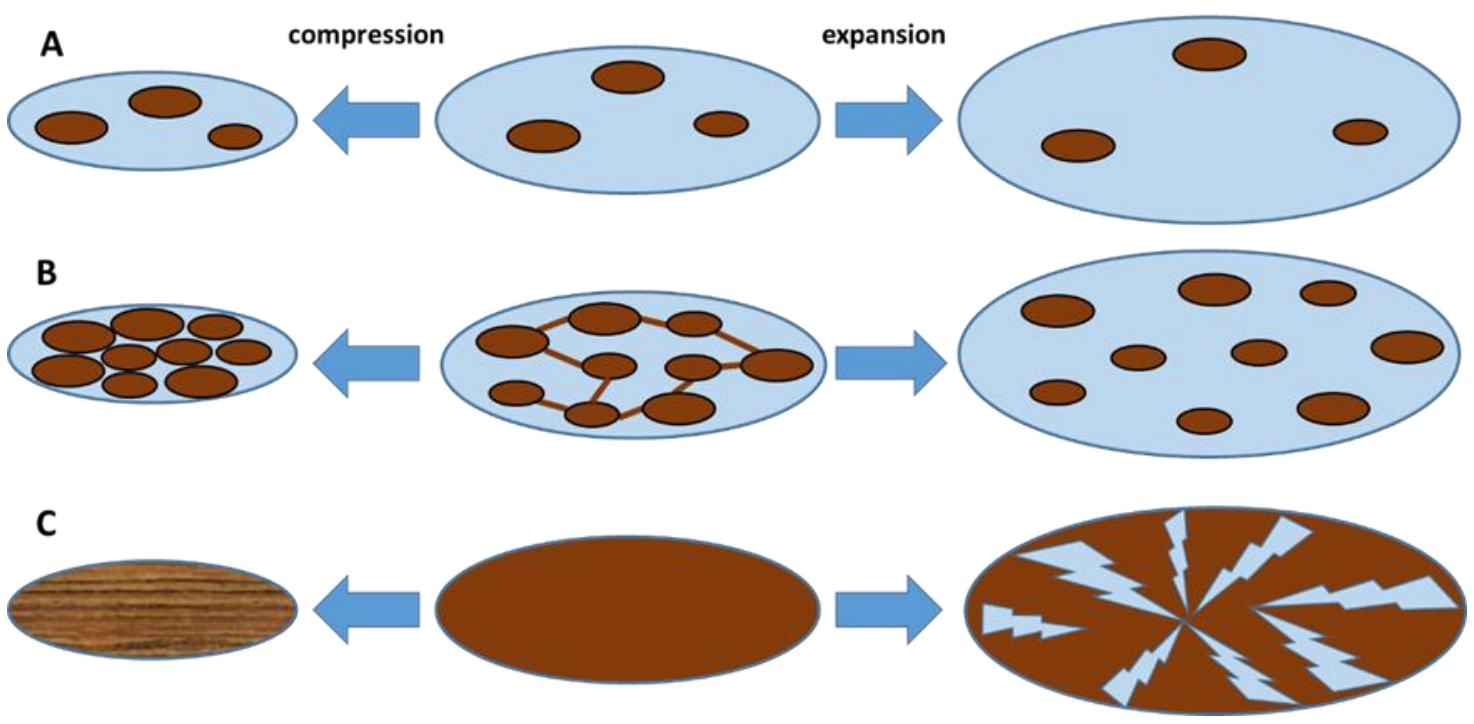

Fig. 9. A scheme of the response of polydopamine films on deformation according to the following initial concentrations of dopamine hydrochloride: $\mathrm{A}-\leq 0.5 \mathrm{~g} / \mathrm{l}$, some domains are formed but do not interact with each other; $\mathrm{B}-0.75-1.5 \mathrm{~g} / \mathrm{l}$, the number and/or size of domains increase and they interact under compression of the surface layer while under expansion the distance between domains is large and they do not interact as probably connections between domains are broken; $\mathrm{C}-\geq 2 \mathrm{~g} / \mathrm{l}$, a continuous homogeneous film is formed which folds under compression and is broken under expansion . 